\title{
Langzeitstudie
}

\section{Jahre länger leben mit intensiver Diabetestherapie}

Eine intensive Diabetestherapie mit niedrigen Blutzucker-, Blutdruckund Blutfettzielen kann das Leben von Typ-2-Diabetikern im Vergleich zu einer konventionellen Behandlung deutlich verlängern - in einer dänischen Studie um acht Jahre.

In den vergangenen 20 Jahren hat sich bei der Diabetestherapie einiges geändert und das zu Recht: Werden nicht nur Blutzucker, sondern auch andere kardiovaskuläre Risikofaktoren konsequent angegangen, übersetzt sich das in eine deutlich höhere Lebenserwartung. Dies lässt sich auch aus einer aktuellen Langzeitauswertung ableiten.

In der 1993 gestarteten Untersuchung Steno 2 wurden 160 dänische Typ-2-Diabetiker mit Mikroalbuminurie in zwei Gruppen eingeteilt, die in Alter, Geschlecht und Albuminausscheidung vergleichbar waren. Die eine Gruppe wurde nach den damals geltenden Leitlinien konventionell und weitgehend bei ihren Hausärzten behandelt, bei den übrigen kümmerte sich eine spezialisierte Diabetesklinik um die Therapie. Zum einen wurde in der Klinik eine Verhaltensänderung versucht, zum anderen sehr zielwertorientiert behandelt - die Patienten sollten bestimmte kardiovaskuläre Richtwerte erreichen.

So wurden in der Gruppe mit konventioneller Behandlung zunächst nur systo-

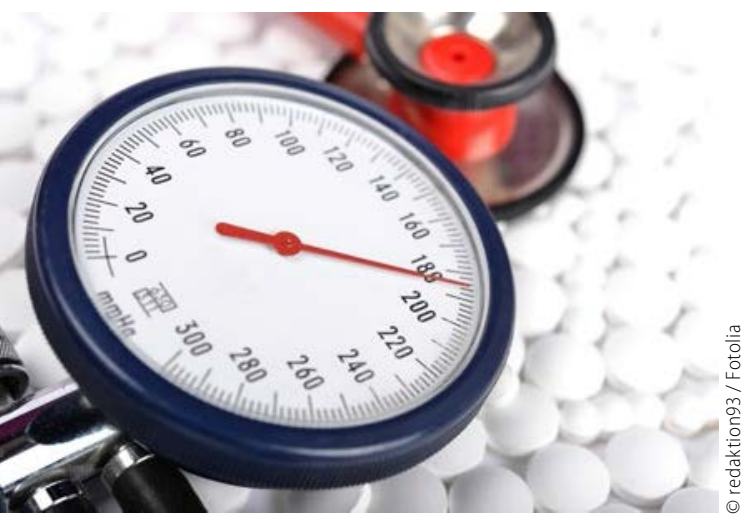

Ein wichtiger Parameter der intensiven Therapie ist die Blutdruckkontrolle. lische Blutdruckwerte unter $160 \mathrm{mmHg}$ angestrebt, in der intensiv behandelten Gruppe waren es 140 mmHg. Der $\mathrm{HbA}_{\mathrm{lc}}$ sollte bei der konventionellen Therapie unter 7,5, bei der intensiven unter 6,5 liegen. Beim Gesamtcholesterin achteten die Ärzte mit konventioneller Behandlung auf Werte unter $250 \mathrm{mg} / \mathrm{dl}$ (6,5 mmol/l), bei intensiver Therapie sollten es weniger als 190 $\mathrm{mg} / \mathrm{dl}$ (4,9 mmol/l) sein. Auch der Triglyzeridzielwert war mit konventioneller Behandlung deutlich höher als mit intensiver (<195 vs. $<150 \mathrm{mg} / \mathrm{dl}$ ). In der Intensivtherapiegruppe bekamen die Patienten zudem ASS bei einer peripheren vaskulären Erkrankung, dies war in der Gruppe mit konventioneller Therapie nicht der Fall.

\section{Deutliche Unterschiede bei \\ Blutdruck, $\mathrm{HbA}_{1 \mathrm{c}}$ und Blutfetten}

Sieben Jahre nach Studienbeginn hatten die Dänen ihre Leitlinien aktualisiert, von da an galten auch für die konventionell Behandelten ähnliche Zielwerte. Die Studienärzte senkten daraufhin die Zielwerte für die intensiv Behandelten noch etwas, letztlich gab es ab dem Jahr 2000 jedoch keine großen Unterschiede mehr bei den Zielvorgaben.

Die Studie endete nach knapp acht Jahren, den Patienten mit der konventionellen Behandlung wurde dann ebenfalls eine intensive Therapie angeboten. $\mathrm{Zu}$ diesem Zeitpunkt gab es in der Tat deutliche Unterschiede zwischen intensiv und konventionell behandelten Patienten beim systolischen Blutdruck (131 vs. 146 mmHg), beim $\mathrm{HbA}_{1 \mathrm{c}}$ (7,9 vs. 9,0) oder Gesamtcholesterin $(4,1$ vs. $5,6 \mathrm{mmol} / \mathrm{l}) \mathrm{so}$ wie einer Reihe weiterer kardiometabolischer Parameter.

Im Jahr 2014 konnte ein Team um Dr. Peter Gæde vom Slagelse Hospital 66 noch lebende Personen ausfindig machen, die an der ursprünglichen Studie teilgenommen hatten. In den 21 Jahren seit Studienbeginn waren 55 Typ-2-Diabetiker (69\%) in der Gruppe mit achtjähriger konventioneller Therapie gestorben, in der Gruppe mit intensiver Behandlung hatte der Tod erst 38 Patienten (48\%) geholt.

Im Median lebten die Diabetiker in der Gruppe mit konventioneller Behandlung noch 13 Jahre, dagegen wurde die mediane Überlebenszeit der Patienten mit intensiver Therapie noch nicht ganz erreicht, hier sind es jedoch bereits acht Jahre mehr.

\section{Seltener mikrovaskuläre Komplikationen}

Ein ähnlicher Unterschied ergab sich bei der medianen Zeit bis zum ersten kardiovaskulären Ereignis oder dem Tod: In der Gruppe mit konventioneller Behandlung trat eines von beiden nach 8,0 Jahren ein, bei intensiver Therapie nach 16,1 Jahren. Insgesamt war die Mortalität unter Berücksichtigung sämtlicher bekannter $\mathrm{Ri}$ sikofaktoren in der Gruppe mit intensiver Behandlung um $45 \%$ reduziert.

Dies ließ sich vor allem auf eine reduzierte kardiovaskuläre Mortalität (minus $62 \%$ ) zurückführen. Bei nicht gefäßbedingten Todesfällen gab es dagegen keine deutlichen Unterschiede. Insgesamt traten kardiovaskuläre Ereignisse in der Gruppe mit intensiver Therapie nur halb so oft auf wie in der mit konventioneller (90 vs. 195). Auch mikrovaskuläre Komplikationen wurden mit intensiver Therapie deutlich seltener beobachtet.

Die Untersuchung, so die Studienautoren, unterstreicht eindrucksvoll den Nutzen der heute weitgehend üblichen intensiven kardiometabolischen Therapie bei Typ-2-Diabetikern mit Mikroalbuminurie.

(Thomas Müller)

GædeP et al. Years of life gained by multifactorial intervention in patients with type 2 diabetes mellitus and microalbuminuria: 21 years follow-up on the Steno-2 randomised trial. Diabetologia 2016; 59:2298-2307 